\title{
Impact of coercive measures on life stories: qualitative study
}

Ingrid Sibitz, Alexandra Scheutz, Richard Lakeman, Beate Schrank, Markus Schaffer

and Michaela Amering

\section{Background}

How people integrate the experience of involuntary hospital admission and treatment into their life narrative has not been explored systematically.

\section{Aims \\ To establish a typology of coercion perspectives and styles of integration into life stories.}

\section{Method}

Transcripts of recorded interviews with 15 persons who had previously been involuntarily admitted to hospital were coded and analysed thematically using a modified grounded theory approach.

\section{Results}

With hindsight, people viewed the experience of involuntary hospital admission as a 'necessary emergency brake', an 'unnecessary overreaction' or a 'practice in need of improvement'. With respect to how they integrated the experience into their life narratives, participants viewed it as 'over and not to be recalled', a 'life-changing experience' or a 'motivation for political engagement'.

\section{Conclusions}

The participants' diverse and differentiated perspectives on coercive measures and their different styles of integration suggest that people may come to accept coercive measures as necessary when confronted with danger to self or others. However, the implementation of coercion needs to be improved substantially to counteract possible long-term adverse effects.

\section{Declaration of interest}

None.
Involuntary admission to hospital and associated coercive practices are relatively common in public mental health services. The concept of coercion varies in the literature. It may refer to the use of physical force or threats of such force, ${ }^{1}$ or the application of more subtle pressure or influence to engender compliance. ${ }^{2}$ Physically forcing people to attend hospital or preventing them from leaving and the use of restraints, seclusion and forced medication are all obvious forms of coercion. Coercion always interferes with a person's autonomy. It is typically justified with reference to the principle of beneficence. Hence, the argument for coercion in mental healthcare depends on the outcome, i.e. the extent to which the good arising from it sufficiently outweighs its inherent harm. Consequently, the outcomes (clinical and social) and the perceptions of the person subjected to coercion must be considered by those who use or seek to justify coercion in psychiatric care.

Empirical evidence relating to the outcomes and impacts of involuntary hospitalisation or treatment remains scarce and inconsistent. ${ }^{3-5}$ Most people who are involuntarily admitted have been found to demonstrate clinical improvement over time, ${ }^{5}$ and mandated out-patient treatment has been found to be associated with improvements in functioning and quality of life. ${ }^{6}$ A small pool of qualitative studies provide data on subjective experiences of involuntary hospital admission. A review of qualitative studies examining different aspects of the experience of coercion highlighted negative themes, such as a sense of violation or abuse of human rights associated with restricted autonomy and limited participation in decision-making; a sense of not being cared for, respected or listened to; and strong negative emotional responses, leading people to feel devalued and stigmatised. ${ }^{7}$ Practices such as restraint and seclusion are often perceived as unnecessary, punishing and harmful rather than therapeutic. ${ }^{8-10}$ Experience of coercion can lead to an internalised sense of self as 'mad and bad', resulting in low self-esteem, stigma and discrimination after discharge. ${ }^{7}$ Compulsory admission and treatment can also have a negative impact on the therapeutic relationship and contribute to a view of hospitalisation and treatment as prejudicial and unjust. ${ }^{11,12}$ However, some people subjected to coercive treatment do come to view it as necessary, and to regard hospital as a place of safety that can offer protection against self-harm, suicide and impulses to harm others. ${ }^{7,8,12}$ Involuntary admission - at least for some - may be a step towards self-awareness and self-reflection and can lead to positive treatment outcomes.

Overall, coercive practices are experienced differently and have both positive and negative aspects. The way people perceive the experience of coercion may profoundly affect their sense of self and identity, which are crucial aspects in the course of illness and recovery. ${ }^{13,14}$ However, up to now there has been no systematic exploration of how people integrate these experiences into a coherent life story. Therefore, this qualitative study of people with a lived experience of involuntary hospital admission and treatment is aimed at establishing a typology of coercion perspectives and styles of integration into life stories.

\section{Method}

\section{Data collection and analysis}

A qualitative design drawing on a modified grounded theory approach, derived from a combination of pragmatism (the doctrine that the meaning of all concepts and actions lies in their observable practical consequences and effects) and Chicago-style interactionism (human beings interpret each other's actions instead of just reacting to them), ${ }^{15}$ was chosen to gain insight into participants' personal beliefs, attitudes and experiences of coercive measures and their impact on life stories. Theoretical sampling and in-depth semi-structured personal interviews were used because previous research suggested a number of areas of interest that needed to be explored. ${ }^{7-9,11,12,16}$ Accordingly, broad open questions were asked relating to the experience of involuntary 
hospitalisation, associated feelings and reactions, how the experience was processed (e.g. through talking to other people), the perceived impact of the experience on the person's life and its personal significance. Semi-structured interviews allowed these areas to be covered, at the same time providing the flexibility to explore emerging themes and individual issues in detail. Questions were open-ended and revised iteratively, allowing a wide range of topics to be explored. The interviews were conducted in the department of psychiatry and psychotherapy at the Medical University of Vienna and lasted between $30 \mathrm{~min}$ and $2 \mathrm{~h}$. All interviews were undertaken face to face by a researcher (A.S.) who had no previous relationship to the participants. Interviews were tape-recorded and transcribed verbatim.

Transcripts were coded and analysed for thematic content inductively. Interviewing and analysis proceeded simultaneously. Three investigators read the first four transcripts, identifying topics that emerged as issues of concern to the participants. These separate readings and the resultant codings were compared and discussed until a consensus was reached. A coding frame was constructed to guide both the conduct of the next interviews and their analysis. Further transcripts were coded, four at a time, independently by the three researchers applying and refining the coding frame. Resulting codes were discussed and checked for consistency and relevance. Transcripts were repeatedly recoded to the refined coding frame and newly emergent themes were explored in subsequent interviews until theoretical saturation was reached. The broad categories included the life story of the respondent, the nature of the illness, experience of coercion, dealing with the experience afterwards, impact of coercion, attitude towards coercion, the role of coercion in the illness and life story of the respondent, and suggestions for improvement (see Appendix). The final coding frame was then systematically applied to all transcripts using QSR NVivo version 7 on Windows (www.qsrinternational.com). This coding frame facilitated the extraction of a typology of perspectives on coercive experiences and styles of integration into the life story to be drawn from the descriptions provided by participants.

\section{Recruitment and participants}

The study commenced after approval from the ethics committee of the Medical University of Vienna. People were invited to participate if they were currently using mental health services, had a history of involuntary commitment and were 18-65 years old. Those who were in an acute psychotic state, presented with a severe thought disorder or had cognitive impairment were not invited to participate. Patients at the Medical University of Vienna department of psychiatry and psychotherapy and at a mental health centre in the county surrounding Vienna who met these criteria were provided with written information about the study. People who were interested in joining the study directly contacted the research team or wrote their telephone number on a list to be contacted. Detailed information about what participation would entail was provided by a researcher. Participants then provided written consent to participate in a taped interview, for the interview to be transcribed and for anonymous quotations to be used in reports and publications.

Seven women and eight men participated in the study before theoretical saturation was reached. Their age ranged from 32 to 66 years (mean 44.6). The majority of participants lived alone $(n=10)$ and only four reported having a current intimate relationship. Their ICD-10 diagnoses included acute psychotic disorder $(n=1)$, schizophrenia $(n=2)$, drug-induced psychosis $(n=1)$, schizoaffective disorder $(n=6)$ and bipolar disorder $(n=5) .{ }^{17}$ The average age at first psychiatric diagnosis was 25.5 years (range 19-40) and the average age at first psychiatric hospital admission was 27.5 years (range 19-57). The mean number of involuntary hospital admissions was 2.1 (range 1-9) and the average time between last involuntary admission and interview was 3.8 years (range 6 months to 9 years).

\section{Results}

The interviews provided rich descriptions of the experience of coercion and associated emotions. All participants provided accounts of physical restraint and/or forced medication when admitted to hospital. People described being in situations in which they felt humiliated, disrespected, helpless and alone. Telling their stories invoked strong emotions which were conveyed in their accounts.

'Being restrained was the most horrible experience I had in my life ... being restrained and not being able to defend yourself and then these applied injections, medication that makes you feel tired, that you want to sleep, but at the same time you are restrained in such a way that you can't fall asleep ... that is horrible.

\section{Perspectives on involuntary admission and coercion}

Three different perspectives on the experience of coercion were identified which accounted for all the variation in these participants' stories. These three perspectives were a 'necessary emergency brake', an 'unnecessary overreaction' and a 'practice in need of improvement'. The same individual could hold different perspectives relating to different admission episodes or aspects of care. For instance, people reported that some involuntary admissions were a 'necessary emergency brake' but others were not (instead being an 'unnecessary overreaction'), or that the admission was necessary but the physical restraint was not. Also, independently of their judgement about the necessity of involuntary admission and treatment, most participants questioned the way coercive measures were applied (a practice in need of improvement). The three perspectives on involuntary hospitalisation and associated coercive measures are described in more detail below.

\section{A necessary emergency brake}

Some participants volunteered that involuntary admission was necessary to maintain their safety or the safety of others. In situations of acute crises, involuntary admission provided a degree of containment and safety not immediately obtainable through other means.

'I was really not well at this time, what else could they do? And, well, it was important, well, and also necessary ... it is an experience that is not absolutely necessary in life, but what I reckon, because I have had this experience in my own life, that it was necessary.'

The need for admission to hospital was often not recognised at the time it occurred, but looking back on events some people concluded that they were not in control and were on a trajectory of destructive behaviour if left unchecked.

'It was the only possibility to bring me back to normality.'

For the brakes to be applied safely, participants highlighted attributes of professionals that were of value such as being respectful, appearing knowledgeable and projecting a sense of being mentally healthy themselves. Some participants saw compulsory hospital admission and indeed the mental health system as part of a social apparatus to ensure the safety and well-being of citizens. These individuals, although not necessarily accepting of interventions at the time, had some faith that those within the system operated with integrity and accepted the expertise of psychiatry in matters relating to mental health.

'If it has to be so. If they don't find anything else, then it has to be so.' 


\section{An unnecessary overreaction}

Sometimes the involuntary hospitalisation was seen as neither helpful nor necessary:

'Three of a total of six involuntary admissions were completely unnecessary.'

Hospitalisation was perceived as a failure to properly identify and address problems and highlighted failures in the system and structures in society for dealing with crisis.

'Again the doctor persuaded me to go to the hospital, and I said actually I don't want to and I believe I could deal with it very well, just with him, outside of the hospital, and then he said I really should go to the hospital and he would also pay the taxi and whatsoever and then I went in again and then the next day I was restrained, but al that would not have happened if I had not gone to the hospital.'

In some instances people perceived that the interventions offered did not help resolve the crisis and in some cases exacerbated problems. Merely providing a safe or containing environment was perceived as unhelpful and did not fulfil the well-expressed needs of the participants. The ways that problems were formulated by mental health staff as psychiatric issues were sometimes contrary to the ways patients saw their problems and what was needed to solve them.

'At that point in time I would have needed only two talks with people before the coercion ... to have the possibility to show the psychiatrist, the police and even my general practitioner where my fears, my paranoia came from . . . I didn't get the possibility to speak with these people, I was instantly put on drugs and my mind was killed.'

Sometimes the need for involuntary admission was accepted but the need for other associated coercive interventions such as physical restraint and forced medication were questioned.

'Looking back, I must say it [involuntary admission] was necessary . . . but if someone would have talked to me, half an hour, all that acute [physical restraint, forced drugs] would not have happened.'

Threats of force were considered unhelpful by everybody, whereas positive pressure and efforts at persuasion were valued.

" "Five nurses are coming to overpower you," that's what he said to me, and for that I blame him ... I mean, he could have tried to persuade me.'

\section{A practice in need of improvement}

Most of the participants, irrespective of their assessment of the need for coercive interventions, complained about the way coercive measures were applied to manage an emergency situation. Coercive interventions were of poor quality, caused harm, left people feeling disrespected or were otherwise in need of improvement. The extent that coercion was required was frequently questioned.

'If it is the first time [the first psychosis] one does not have a relationship with a good doctor with whom a trustful relationship could have been developed and from whom treatment would be accepted, well, and that's the next thing, "treatment", that is as if I would treat an object, yes, but a human should not be treated, instead, I should act with them and develop together new strategies which lead to improvement.

Participants provided numerous suggestions for attenuating the impact of involuntary hospitalisation and suggested more helpful responses to crisis situations. For example, some suggested providing a place or room for people experiencing psychosis in which they could be accompanied by health professionals who were able to be with them, and enable them to feel safe without resorting to coercive practices. The importance of ongoing provision of information about therapy and further treatment was emphasised as a means to allay people's fears and ensure that patients felt secure during intense experiences.

'I got no information about the injection and what it contained, probably in my situation it would have also been adequate if they had just said, "dear Ms A., there is danger that you might jump out of the window if God tells you to do so, so listen, you have been brought here with the police, we are in a hospital here and you have to stay for observation". ... then they could have saved themselves the trouble of the injection, because that way it would have been possible to talk to me.'

Lack of information about what was to happen next, and the lack of a clear rationale for treatment as well as coercive measures contributed to increased anxiety and sometimes a worsening of psychotic experiences.

and then an injection and if you are confused anyway, in my opinion this can make the situation even worse.

Some participants suggested that specialist, dedicated crisis intervention teams might reduce the amount of involuntary treatment and enhance trust in the mental health system.

'I know this example about a team that intervenes in situations of crises, a good team, that convinces, that the police is not needed to convince people to go to the hospital and there are data saying that people recover faster after that, and it would pay to have such a team, with trust, and they explain why it is necessary to go to the hospital.'

The need for more ordinary conversation with health professionals was emphasised by the participants, many of whom experienced mental health staff as being aloof and unavailable.

'This would be interesting, if such a conversation would help in any way. When I get in such a situation again, I wish to have someone who talks to me.

Participants highlighted the importance of having access to helpers whom they were able to trust, but conceded that trust was something that evolved over time and was compromised by coercion. People considered that their problems were meaningful and that health professionals should recognise and respond to the unique content and circumstances associated with the crisis.

'To help people to understand the key to psychosis, each psychosis has a message.'

\section{Integration of experiences into life stories}

Three different styles of integrating the experience into the participant's life story were differentiated.

\section{Over, not to be recalled}

A few participants regarded their involuntary admission as an exceptional event which was now over. They did not want to be reminded of it and did not anticipate such an event happening again. Despite participating in the research, they were reluctant to think about the event and avoided potentially painful reminders of that period of their lives.

'I don't know it [the impact of coercive measures] any more and I don't talk about it.'

'I don't want to talk about or remember it, and when I realise in a dialogue that the other person is affected as well and might find it burdensome, then it is even worse

usually I don't think about it any more because I don't want to remember, same with regular psychiatric hospitalisations.'

\section{A life-changing experience}

Most participants emphasised that their life had changed after involuntary admission and treatment. They experienced changes in the following areas.

Impact on self-esteem and sense of self. Having experienced coercive measures can have detrimental effects on self-esteem:

'It leads to an absolute inferiority complex, I have the feeling that I am not worth talking to other people, already thinking that I am not worth it, well, we can say destroying my personality.'

The sense of vulnerability to being out of control and being subjected to the control of others contributed to a profound uncertainty in the sense of self and fear of coercion being exercised again:

'The consciousness that there is lack of control, that you cannot control anything, that you cannot control yourself, you cannot decide yourself . . . I always face it that something happens again.'

'It is the moment of breakdown, you have the feeling that you cannot get out. I think that I could get in any time again.

Some engaged in lengthy periods of psychotherapy particularly focusing on the experience of hospitalisation: 
'Well, then I was in psychotherapy, I was there for one and a half years and we processed all that. Yeah, it often was not that easy, because to speak about such a thing is not easy at all.'

Impact on relationships and community life. After involuntary admission people lived with a sense of being under surveillance by their family and friends and therefore felt inhibited in expressing themselves freely. This led to a 'life on probation' and a sense of a changed identity:

'I often thought, this can't be true, I can't do anything, I can't go anywhere, can't show any kind of personality, the first thing that happens is that I have to go to a psychiatric clinic.'

People reported a sense that others treated them differently from before; they were no longer considered as credible, reliable or a full social participant:

'Because of the involuntary admission the judge was convinced in his decision that there is something not quite right with me and that my son was right."

Participants reported becoming more cautious in their interactions with people, were sensitive to cues of rejection and judgement, and perceived that others did not wish to hear about their experiences:

'At the beginning, after discharge from the psychiatric hospital, I talked a lot about what happened to me but then I changed, I kept quiet about it, because most people can't deal with it, they actually don't understand it.

This constellation of response to coercion extended to a general distrust of others, particularly of medical professionals:

'Yeah, for sure I became more cautious related to doctors and nurses.'

For some, involuntary admission to hospital had tangible negative legal consequences:

'I don't find it good that it is on record . . . I can't get life insurance . . . and my driving licence is limited in time and to prolong it costs a lot of money.'

Impact on health. Coercive interventions were perceived as contributing to prolonged hospital stays, negative effects on employment and social roles, and poor recovery.

'Well, I must say, that all that [coercion] impacts the length of treatment, the amount of drugs, then the illness can be pushed, aggravated, the dose of drugs gets higher if you have to deal with injustices in hospital ... if they don't believe you then the subsequent depression is deeper, you get into work more slowly, you get into recovery more slowly ... the traumatic experiences can contribute to a chronic state [of illness].'

'My life changed that way that for a long time I gave up every aim, didn't see any aims, didn't want to achieve anything in life any more.

Positive changes. Some positive changes were also acknowledged. People suggested that their experience of bad times helped them to gain an appreciation of their current stable situation, more consciously enjoy everyday life and be more relaxed when confronted with adversity:

'Sometimes I think I really felt bad often but now I'm well again and then in a sense appreciate that I had this experience, because if I hadn't had this experience then couldn't enjoy it so consciously that I'm well at the moment.

'Actually I became more easy-going and some things I view more easily, if you have overcome such an illness you look differently on things in life.

Some participants emphasised that they took their illness more seriously and tried to prevent further coercive interventions:

'I changed the doctor . . . that eventually I come in voluntarily or that I increase the amount of drugs and to have an advanced directive.'

Respondents also mentioned that their experience led to a better understanding of psychiatric patients and more vulnerable people in general:

'The experience is not just negative, because that way I got a heart for vulnerable people.

Sometimes life changes following the involuntary admission could not be distinguished from life changes following the psychiatric illness:

'I believe that [it] happens not just because of the experience of coercion, the illness itself changes [people] a lot ... suddenly all is different.

\section{Motivation for political engagement}

The experience of being coerced and experiencing the psychiatric system as poor provided an impetus to become involved in seeking reform of the system. Some people became politically active and through various means sought to draw public attention to the negative consequences of coercion and amending laws that permitted psychiatric coercion.

"I said, "somewhere down the road, I will publicly take them to court" ... all of the hospitals, some of the doctors who treated me against my will and didn't want to hear me... not to seek revenge but to draw attention to this issue and in light of the future for many other psychiatric users, who could experience the same thing, not being heard like I was.

People wanted mental health professionals to know how service users experience coercion and they sought to find ways to engage with health professionals on a different footing to do so. People hoped that discussions with professionals, carers and the public might help to improve psychiatric services and reduce negative societal and social consequences associated with psychiatry and involuntary treatment:

'I attended a lot of seminars at the social academy where users can speak about the way they see their illness and I was also asked to become a lecturer in education and training for police and paramedics.

Some people became engaged in activities with other service users or former service users to improve their situation and the situation of others. They emphasised the importance of the exchange of experiences and peer support in their own recovery: 'The exchange of experiences, how others experienced it and how they dealt with it, especially how they deal with it now in their life.

\section{Discussion}

This study set out to explore the views and recollections of people who had experienced involuntary hospital admission. The analysis of participants' opinions and comments about involuntary treatment revealed that people had diverse and differentiated perspectives about involuntary admission and associated coercive interventions. Participants regarded their admission as a 'necessary emergency brake' or as an 'unnecessary overreaction', and sometimes one and the same person reported that some involuntary hospitalisations were necessary whereas others were unnecessary. Some people regarded their treatment in hospital as necessary but forced medication and physical restraints as unnecessary. These different perspectives on involuntary hospital admission and treatment have been noted in the literature. . $^{3,5,18,19}$ However, it has rarely been acknowledged that these different perspectives may be held by the same individual, and that people develop a nuanced, reflective and differentiated view. Although it was regarded as necessary in case of acute crisis and lack of control, it was judged as unnecessary or excessive reaction in other situations when listening and understanding on the part of staff could have contributed to de-escalating the situation.

Many participants considered practices associated with involuntary hospital admission to be 'in need of improvement'. Independently of whether they regarded these practices as necessary or unnecessary, they agreed that involuntary commitment was acceptable in cases of real danger to oneself or to others but were critical of the way in which coercive measures involving forced medication and restraints were actually implemented. A strong plea for information and orientation, communication and respect, and the need to strive to communicate about and possibly understand the content of psychotic experience was made. These aspects are in accord with previous studies, ${ }^{7-10}$ which concluded that the negative effects of compulsory treatment can be greatly attenuated through 
respectful engagement with health professionals, not overextending coercive processes, maintaining usual routines, rituals and relationships while under involuntary care, and a sense of being cared for or kept safe.

\section{Styles of integration and effects of admission}

Three different styles of integrating the experience of coercive measures into the life story were found. Some people regarded their involuntary admission as an exceptional event and did not want to be reminded of it; most people clearly indicated that the event had changed their life. Coercive measures violated the individual's integrity, contributed to low self-esteem and led to a sense of vulnerability. In accordance with Wynn's finding that people who had been subjected to coercive measures were afraid of being subjected to coercive measures again, ${ }^{12}$ people in our study reported entering a 'life on probation'. They were eyed by others with scepticism and tried to change their own behaviour to reduce the likelihood of involuntary admission in the future. People also changed their views towards life and towards other people, and in many cases it was harder for them to trust others, especially medical doctors. This indicates that experiences of involuntary hospital admission and treatment can undermine the therapeutic relationship and people's trust in mental health services, causing them to avoid such services. Distrust and other negative effects on health such as resignation and purposelessness hinder the recovery process. Such possible negative consequences of coercion have also been reported in other studies. ${ }^{11,12,20-23}$

Participants in this study reported stigma and discrimination after involuntary admission. They felt treated differently by others, that they were no longer considered as credible and reliable, and they perceived that their friends and relatives did not want to speak or hear about their involuntary admission and in some instances avoided them. People with experience of coercion may be especially exposed to stigmatising and discriminating behaviour in the community - an assumption supported by a recently published study by Thornicroft $e t$ al, who found a greater amount of negative discrimination among participants who had been treated compulsorily. ${ }^{24}$

Some positive changes following involuntary admission were also mentioned. They related mainly to appreciating and enjoying the current stable situation after experiencing a difficult time. Interestingly, nobody regarded the involuntary admission itself as a positive, life-saving event that contributed to reintegration into society and reconnection with family and friends. A possible explanation, besides the stigma and shame associated with such an event of loss of control, could be that people had not only to deal with life changes following the involuntary admission but also with far-reaching life changes following the psychiatric illness, which sometimes was not easy to distinguish. For some people the experience of coercion was a 'motivation for political engagement'; they felt the necessity to raise public awareness of the negative consequences of coercion and to improve the situation for service users. In this study, more negative than positive aspects of coercion were reported and included long-term detrimental consequences such as being more insecure, anxious and mistrustful, which represent negative effects on sense of self and self-assurance. Although our study may have had a selection bias towards a more negative view, Priebe et al in a survey of 396 patients from consecutive involuntary hospital admissions in England found that $60 \%$ considered their admission unjustified a year later. ${ }^{18}$ Given that one might expect greater insight with the passage of time, this brings the helpfulness of involuntary hospitalisation into question.

\section{Study limitations}

Further limitations of this study need to be considered. Using a qualitative design makes it possible to obtain in-depth views of a variety of different perspectives on coercion, and the different ways of integrating such an event into one's life story, but it is not possible to say how often these viewpoints occur for others. The sample contains participants who were interested in the topic and wanted to be interviewed. Other perspectives and ways of integration could occur among people who might feel less inclined to speak about the experience of coercion. Another point is the time elapsed between the event of hospital admission and the research interview. In contrast to previous qualitative studies of experiences of coercion, ${ }^{7-12}$ which included patients who had recently been subjected to coercive measures, this study included participants who had a history of involuntary admission with the last of these being between 6 months and 9 years ago. Although memory bias might have impaired the recall of experiences, there had been time to reflect on the event and long-term consequences could be reported.

\section{Implications for mental health practice}

Involuntary hospital admission and coercive measures to prevent harm are retrospectively acceptable to at least some of those subjected to such measures providing they are implemented with respect, not extended beyond the prevention of harm and undertaken in a climate of trust, information-sharing, genuine interest and understanding. Coercion may be regarded as unnecessary and even harmful. Therefore the use of coercive measures ought to be confined to acute crisis events and implementation should be improved substantially. Education and service development should focus on both how to prevent coercion and how to apply coercive measures in case of acute danger. The integration of the views of service users is crucial to make essential improvements. Indeed, service user involvement in every aspect of individual treatment decision-making is the ideal.

The views of the participants of this study might also help other service users to cope with coercive measures. At least for some people, it is important to have the possibility of speaking about the experience with family, friends, professionals and other service users. Professionals might incorporate discussing the experience of being coerced in formal group therapy, and similar benefits might arise from service users participating in self-help groups in which these experiences might be safely discussed.

\footnotetext{
Ingrid Sibitz, MD, Department of Psychiatry and Psychotherapy, Medical University of Vienna, and Ludwig Boltzmann Institute for Social Psychiatry, Vienna; Alexandra Scheutz, MD, Department of Psychiatry and Psychotherapy, Medical University of Vienna, Austria; Richard Lakeman, DNSc, School of Nursing, Dublin City University, Dublin, Ireland; Beate Schrank, MD, Markus Schaffer, MD, Department of Psychiatry and Psychotherapy, Medical University of Vienna; Michaela Amering MD, Department of Psychiatry and Psychotherapy, Medical University of Vienna, and Ludwig Boltzmann Institute for Social Psychiatry, Vienna, Austria
}

Correspondence: Dr Ingrid Sibitz, Medical University of Vienna, Department of Psychiatry and Psychotherapy, Währinger Gürtel 18-20, A-1090 Vienna, Austria. Email: ingrid.sibitz@meduniwien.ac.at

First received 29 Sep 2010, final revision 25 Jan 2011, accepted 17 May 2011

\section{Acknowledgements}

We thank the patients who served as participants in this project. We gratefully acknowledge Wolfgang Grill, Community Mental Health Centres, Psychosoziale Zentrum $\mathrm{GmbH}$, Stockerau, Austria, for his help with the recruitment of study participants. 


\section{Appendix}

\section{Text coding categories}

1. Life: the life story of the person.

2. Own illness: symptoms, causes, treatment and coping.

3. Experience of coercion: preconditions, physical force, forced treatment, communication with mental health professionals, people involved in the process of involuntary admission, evaluating the experience, reacting and responding to coercive measures.

4. Dealing with the experience afterwards: repression, reflection, processing, activities, evaluation/closure (marking/framing).

5. Impact of coercion: effects on the person and on recovery, social and legal consequences.

6. Attitude towards coercion: the necessity, or lack thereof, of coercive measures.

7. The role of coercion: the role of coercion in the illness and life story of a person.

8. Suggestions for improvement: general and specific ideas about desired changes in psychiatric practice.

\section{References}

1 Macklin R. Man, Mind and Morality: The Ethics of Behavior Control. Prentice-Hall, 1982.

2 Lutzen K. Subtle coercion in psychiatric practice. J Psychiatr Ment Health Nurs 1998; 5: 101-7.

3 Wynn R. Coercion in psychiatric care: clinical, legal and ethical controversies. Int J Psychiatry Clin Pract 2006; 10: 247-51.

4 Sailas E, Fenton M. Seclusion and restraint for people with serious mental illnesses. Cochrane Database Syst Rev 2000; 2: CD001163.

5 Katsakou C, Priebe S. Outcomes of involuntary hospital admission - a review. Acta Psychiatr Scand 2006; 114: 232-41.

6 Link B, Castille DM, Stuber J. Stigma and coercion in the context of outpatient treatment for people with mental illnesses. Soc Sci Med 2008; 67 409-19.

7 Katsakou C, Priebe S. Patients' experiences of involuntary hospital admission and treatment: a review of qualitative studies. Epidemiol Psichiatr Soc 2007; 16: $172-8$.
8 Holmes D, Kennedy SL, Perron A. The mentally ill and social exclusion: a critical examination of the use of seclusion from the patient's perspective Issues Ment Health Nurs 2004; 25: 559-78.

9 Kuosmanen L, Hatonen $\mathrm{H}$, Malkavaara H, Kylma J, Valimaki M. Deprivation of liberty in psychiatric hospital care: the patient's perspective. Nurs Ethics 2007; 14: 597-607.

10 Olofsson B, Norberg A. Experiences of coercion in psychiatric care as narrated by patients, nurses and physicians. J Adv Nurs 2001; 33: 89-97.

11 Thogersen $\mathrm{MH}$, Morthorst $\mathrm{B}$, Nordentoft $\mathrm{M}$. Perceptions of coercion in the community: a qualitative study of patients in a Danish assertive community treatment team. Psychiatr Q 2010; 81: 35-47.

12 Wynn R. Psychiatric inpatients' experiences with restraint. J Forens Psychiatry Psychol 2004; 15: 124-44.

13 Estroff SE. Self, identity, and subjective experiences of schizophrenia: in search of the subject. Schizophr Bull 1989; 15: 189-96.

14 Davidson L, Strauss JS. Sense of self in recovery from severe mental illness Br J Med Psychol 1992; 65: 131-45.

15 Corbin J, Strauss A. Basics of Qualitative Research, 3rd edn. Sage, 2008.

16 Gilburt $H$, Rose $D$, Slade $M$. The importance of relationships in mental health care: a qualitative study of service users' experiences of psychiatric hospital admission in the UK. BMC Health Serv Res 2008; 8: 92.

17 World Health Organization. The ICD-10 Classification of Mental and Behavioural Disorders. Clinical Description and Diagnostic Guidelines. WHO, 1992

18 Priebe S, Katsakou C, Amos T, Leese M, Morriss R, Rose D, et al. Patients views and readmissions 1 year after involuntary hospitalisation. $\mathrm{Br} J$ Psychiatry 2009; 194: 49-54.

19 Priebe S, Katsakou C, Glöckner M, Dembinskas A, Fiorillo A, Karastergiou A, et al. Patients' views of involuntary hospital admission after 1 and 3 months: prospective study in 11 European countries. Br J Psychiatry 2010; 196: $179-85$

20 O'Hagan M. Force in mental health services: international user/survivor perspectives. Mental Health Practice 2004; 7: 12-7.

21 O'Brien AJ, Golding CG. Coercion in mental healthcare: the principle of least coercive care. J Psychiatr Ment Health Nurs 2003; 10: 167-73.

22 Amering M, Schmolke M. Recovery in Mental Health: Reshaping Scientific and Clinical Responsibilities. Wiley, 2009

23 Link BG, Struening EL, Neese-Todd S, Asmussen S, Phelan JC. Stigma as a barrier to recovery: the consequences of stigma for the self-esteem of people with mental illnesses. Psychiatr Serv 2001; 52: 1621-6.

24 Thornicroft G, Brohan E, Rose D, Sartorius N, Leese M. Global pattern of experienced and anticipated discrimination against people with schizophrenia: a cross-sectional survey. Lancet 2009; 373: 408-15. 